This article is forthcoming in The Lancet Psychiatry. Please quote from the published version.

Reference: Nelson, Barnaby, Patrick D. McGorry, and Anthony Vincent Fernandez. Forthcoming. "Integrating Clinical Staging and Phenomenological Psychopathology to Add Depth, Nuance, and Utility to Clinical Phenotyping: A Heuristic Challenge." The Lancet Psychiatry.

\title{
Integrating clinical staging and phenomenological psychopathology to add depth, nuance and utility to clinical phenotyping: A heuristic challenge
}

Barnaby Nelson ${ }^{1,2}$

Patrick D. McGorry ${ }^{1,2}$

Anthony Fernandez $z^{3,4}$

Lancet Psychiatry - Personal View

Corresponding author: B Nelson, Orygen, 35 Poplar Rd, Parkville, Victoria 3052, Australia; nelsonb@unimelb.edu.au; +61 393422800.

${ }^{1}$ Orygen, Parkville, Victoria, Australia (Prof B Nelson, PhD, Prof P McGorry, MD)

${ }^{2}$ Centre for Youth Mental Health, The University of Melbourne, Parkville, Victoria, Australia (Prof B Nelson, PhD, Prof P McGorry, MD)

${ }^{3}$ University of Oxford, United Kingdom (A Fernandez, PhD)

${ }^{4}$ Kent State University, Kent, Ohio, USA (A Fernandez, PhD) 


\section{Abstract}

Psychiatry has witnessed a new wave of approaches to clinical phenotyping and the study of psychopathology, including the National Institute of Mental Health's Research Domain Criteria, clinical staging, network approaches, the Hierarchical Taxonomy of Psychopathology, and the general psychopathology factor, as well as a revival of interest in phenomenological psychopathology. The question naturally emerges as to what the relationship between these new approaches is - are they mutually exclusive, competing approaches, or can they be integrated in some way and used to enrich each other? In this opinion piece, we propose a possible integration between clinical staging and phenomenological psychopathology. Domains identified in phenomenological psychopathology, such as selfhood, embodiment, affectivity, etc., can be overlaid on clinical stages in order to enrich and deepen the phenotypes captured in clinical staging ('high resolution' clinical phenotypes). This approach may be useful both ideographically and nomothetically, in that it could complement diagnosis, enrich clinical formulation, and inform treatment of individual patients, as well as help guide aetiological, prediction, and treatment research. The overlaying of phenomenological domains on clinical stages may require that these domains are reformulated in dimensional rather than categorial terms. This integrative project requires assessment tools, some of which are already available, that are sensitive and thorough enough to pick up on the range of relevant psychopathology. The proposed approach offers opportunities for mutual enrichment: clinical staging may be enriched by introducing greater depth to phenotypes; phenomenological psychopathology may be enriched by introducing stages of severity and disorder progression to phenomenological analysis. 
In a recent publication, Maj (1) argues that in psychiatry, in contrast to other medical specialities, the information conveyed by diagnosis is insufficient in itself for therapeutic and prognostic purposes. Maj argues that there is, therefore, a pressing need for a more detailed clinical characterisation of the individual case and that the field should start to promote the construction and validation of tools that systematically guide the clinician and researcher in this characterisation. The field has witnessed a new wave of approaches to researching psychopathology, including the National Institute of Mental Health (NIMH)'s Research Domain Criteria (RDoC), clinical staging, network approaches, the Hierarchical Taxonomy of Psychopathology (HiToP), and the general psychopathology ('P') factor, as well as a revival of interest in phenomenological psychopathology. Several of these approaches have been presented as "alternatives" to the DSM/ICD approach to psychiatric diagnosis, on the grounds that they have more robust validity and utility in aetiological research and in guiding treatment decision-making (1-3). Maj, however, argues that they are in fact unlikely to replace current diagnostic practices, but may be able to complement current diagnoses by significantly improving clinical characterisation of individual cases and groups of patients (i.e., clinical phenotyping).

The question naturally emerges as to what the relationship between these new approaches is - are they mutually exclusive, competing approaches, or can they be integrated in some way and used to enrich each other for the purpose of more 
detailed and useful clinical phenotyping? In this opinion piece, we propose a possible integration between clinical staging and phenomenological psychopathology. While the focus here is on exploring the possibility of integrating these two approaches, a similar question could be asked with regard to the other approaches (e.g., the relationship between $\mathrm{RDOC}$ and HiTOP). We argue that this integration has two key advantages for clinical staging: 1) it adds depth and nuance to stage-based clinical phenotypes; 2) it facilitates both idiographic and nomothetic understanding of the condition in question (i.e., an understanding of the particular patient and of the more general condition). To explain this proposal, we briefly outline the clinical staging model and the approach of phenomenological psychopathology, particularly how it can be applied in dimensional terms, before turning to their potential integration.

In brief, clinical staging attempts to determine the position of an individual along a continuum of illness, defined according to stages: Stage $0=$ no current symptoms, Stage $1 \mathrm{a}=$ help-seeking with distress, Stage $1 \mathrm{~b}=$ attenuated (i.e., sub-threshold) syndrome, Stages 2-4 = full threshold disorder with varying degrees of recurrence and severity (see Figure 1a for illustration)(3-5). While criteria can certainly be used to define these clinical stages [e.g., see (6-9)], part of the clinical appeal of the staging model is that clinical stage can be readily judged based on symptom severity and extension. Clinical staging takes a quasi-dimensional approach to symptomatology; it delineates step changes on top of continuous transdiagnostic symptomatology to guide treatment decision-making, prediction, and aetiological research. As illustrated in Figure 1 (panel A), clinical staging takes a transdiagnostic approach by delineating illness stages across symptom domains (psychosis, mood, anxiety symptoms, etc.), rather than being wed to traditional diagnostic categories. This accommodates the 
mixed clinical presentations and high levels of comorbidity frequently seen, particularly in the early stages of disorder, as well as fluctuating and heterotypic clinical trajectories (e.g., a Stage $1 \mathrm{~b}$ clinical presentation characterised by anxiety and mood symptoms evolving into a Stage 2 disorder with prominent psychotic symptoms) $(3,10,11)$. In contrast to the $D S M / I C D$, clinical staging, in principle, allows for personalised treatments based on specific combinations of symptoms and their degree of severity and differentiation (e.g., while a Stage $1 \mathrm{~b}$ patient with attenuated psychotic symptoms and mood symptoms might be treated with cognitive-behaviour therapy and an antidepressant medication, a Stage 2 patient with predominant psychotic symptoms might be treated with an antipsychotic medication and psychosocial support). However, while the concepts behind clinical staging are sound, its success requires that symptoms are clearly described and defined. In other words, the personalised interventions and advances in prediction and aetiological research promised by clinical staging are likely to require 'high resolution' capture of psychiatric symptoms and signs, i.e., phenotypic depth and nuance (12). We argue that this phenotypic depth and nuance can be provided by phenomenological psychopathology.

Emerging from the philosophical tradition of phenomenology, phenomenological psychopathology is an interdisciplinary research program that describes and classifies experiential disturbances in mental disorders (i.e., characteristic features of the experience and expression of mental disorders)(13)[see Appendix, p.2]. Phenomenological psychopathologists often operate within a categorial framework, articulating the experiential disturbances characteristic of, for example, bipolar disorder or schizophrenia. However, many phenomenological psychopathologists 
share psychiatry's scepticism about current diagnostic categories (13). Some of these researchers now use phenomenology to distinguish experiential disturbances both within and across traditional diagnostic categories. In contrast to the DSM/ICD approach, and arguably also new approaches to clinical characterisation such as RDoC and HiToP, phenomenological psychopathology is based on well-articulated accounts of 'normal' or 'healthy' experience. So, rather than start an investigation from a DSM/ICD category, these research studies typically start from basic, elemental features of human subjectivity that have been identified in the phenomenological tradition. These overlapping features are thought of as constituting or making up conscious life. Some of the structures of experience commonly studied by phenomenological psychopathologists are: 1. selfhood, including the tacit sense of ownership and agency of one's experience and behaviour, as well as the narrative construction of a social identity; 2 . intersubjectivity, including the capacity to perceive and engage with other human beings as expressive subjects, rather than mere objects; 3. embodiment, including the capacity to shift between experiences of one's body as a power of engaging with the world (Leib) and as a material object (Körper); 4. affectivity, including resonance with and attunement to one's environment through moods and emotions; 5 . understanding, including the way one tacitly interprets and makes sense of everyday objects and environments; 6 . temporality, including the tacit anticipation and retention of experiences, which constitutes the lived flow of time; and 7. spatiality, including one's capacity to appreciate and operate within different normative spaces, such as the space of the home, the theatre, the classroom, and so on. The term 'existential structures' is used to refer to these features because they are thought to structure or organise our experience (14-16)[see Appendix, p.1]. 
Each existential structure has a diverse range of states in which they might be for a given individual at a given time. For example, the existential structure of affectivity may be expressed in anxiety, boredom, or joy; the existential structure of temporality may be expressed in eager anticipation or slowly whiling away the time. Moreover, existential structures may undergo more fundamental disturbances. For example, a person may partially lose the capacity to be affectively attuned through moods; or, in the case of temporality, a person may undergo a fundamental disturbance in their capacity to retain and anticipate experiences. Typically, phenomenological psychopathologists identify an existential structure (or set of existential structures) to frame their study, but they aim to describe the particular way in which it manifests in the condition in question. For instance, Martin and colleagues (17) examined bipolar disorder through the lens of temporality and focus on the particular ways in which the experience of time is disturbed in this disorder and how these disturbances may negatively impact insight and reasoning in manic episodes.

The existential structures can form domains for dimensional phenomenological research, which would in some ways be analogous to the RDoC domains (see (14, 18) for full discussion of this proposal). In recent years, instruments have been developed [e.g., the Examination of Anomalous Self Experience (EASE)(19) and the Examination of Anomalous World Experience (EAWE)(20)] that facilitate this approach and provide quantitative metrics of the type and severity of phenomenological disturbances. The domains can be divided into constructs and sub-constructs. For instance, the domain of selfhood is commonly divided into the constructs of basic self (a pre-reflective, tacit level of selfhood) and narrative self 
(personality, social identity, habits, style, personal history, etc.). The basic self might be further organised into the subconstructs of cognition and stream of consciousness, self-awareness and presence, bodily experiences, demarcation of self-world boundary, and existential orientation, as captured in the sections of the EASE instrument. A phenomenological study would then investigate, with respect to a particular patient or class of patients, the experiential disturbances within each of these sub-constructs.

Let us illustrate this approach by briefly summarising two recent studies. Sass and Pienkos (21) studied selfhood across schizophrenia, melancholia and mania. They found that self-world boundaries were unstable across the conditions examined, but in fundamentally different ways: heightened in melancholia, diminished in mania and schizophrenia but the former with an ecstatic or benign mood tone and the latter with solipsism and erosion of first-person perspective (21). In this case, the findings supported current diagnostic boundaries. Ratcliffe (22), in contrast, studied temporality in major depressive disorder. He found that temporal experience was disturbed in quite different ways within depression. Patients varied on single or multiple constructs/subcontracts of loss of drive/momentum, loss of futural projects, and loss of futural significance. These findings suggest profound heterogeneity within the diagnostic category of major depressive disorder and provide preliminary evidence for subtypes within this category (22). These types of investigations adopt a dimensional research framework by analysing the specific ways in which a particular phenomenological domain ('existential structure') might be disturbed across and within diagnostic categories (i.e., the severity and specific features of this disturbance). By putting diagnostic boundaries temporarily to one side, using this lens 
of analysis might refract certain disturbances of existential structures (e.g., selfhood, temporality) that are shared across conditions, highlight distinctions between conditions, or identify subtypes within a particular diagnostic category.

The crux of the current proposal is that this dimensional phenomenological analysis of psychopathology could be overlaid on top of clinical stages. That is, disturbances of existential structures, and their constructs and subconstructs, could be analysed according to disorder severity and stage progression. Figure 1 provides a heuristic illustration of how this approach might be pursued. While panel A presents the clinical staging model as it currently stands, panel B shows heuristic examples of how disturbances of particular existential structures might evolve across stages of disorder. These patterns or trajectories across stages might vary by individual patient and by type of disorder; variation of type of disorder is illustrated in this animation: https://s6.gifyu.com/images/animation-40b881647ef7dcebb.gif. In this illustration, for example, disturbances of selfhood are particularly characteristic of schizophrenia spectrum disorders and become more pronounced as stages of disorder become more advanced (see (23) for preliminary empirical support for this relationship between disturbances of selfhood and stage of disorder, particularly over early stages). This analysis can be nuanced by examining constructs within the domain of selfhood. Current evidence, for example, suggests that the construct of basic self is particularly relevant to schizophrenia spectrum disorders while the construct of narrative self is more relevant to borderline personality disorder (24-26). Exactly how this relationship between existential structure and syndrome relates to or develops across stages of mental disorder is an empirical question invited by this proposed integrative model. 
Figure 1 Text: Heuristic diagram of how existential structures emerging from phenomenological psychopathology can be overlaid on top of clinical stages. Panel A shows the clinical staging model as it currently stands. Panel B shows heuristic examples of how disturbances of particular existential structures might evolve across stages of disorder.
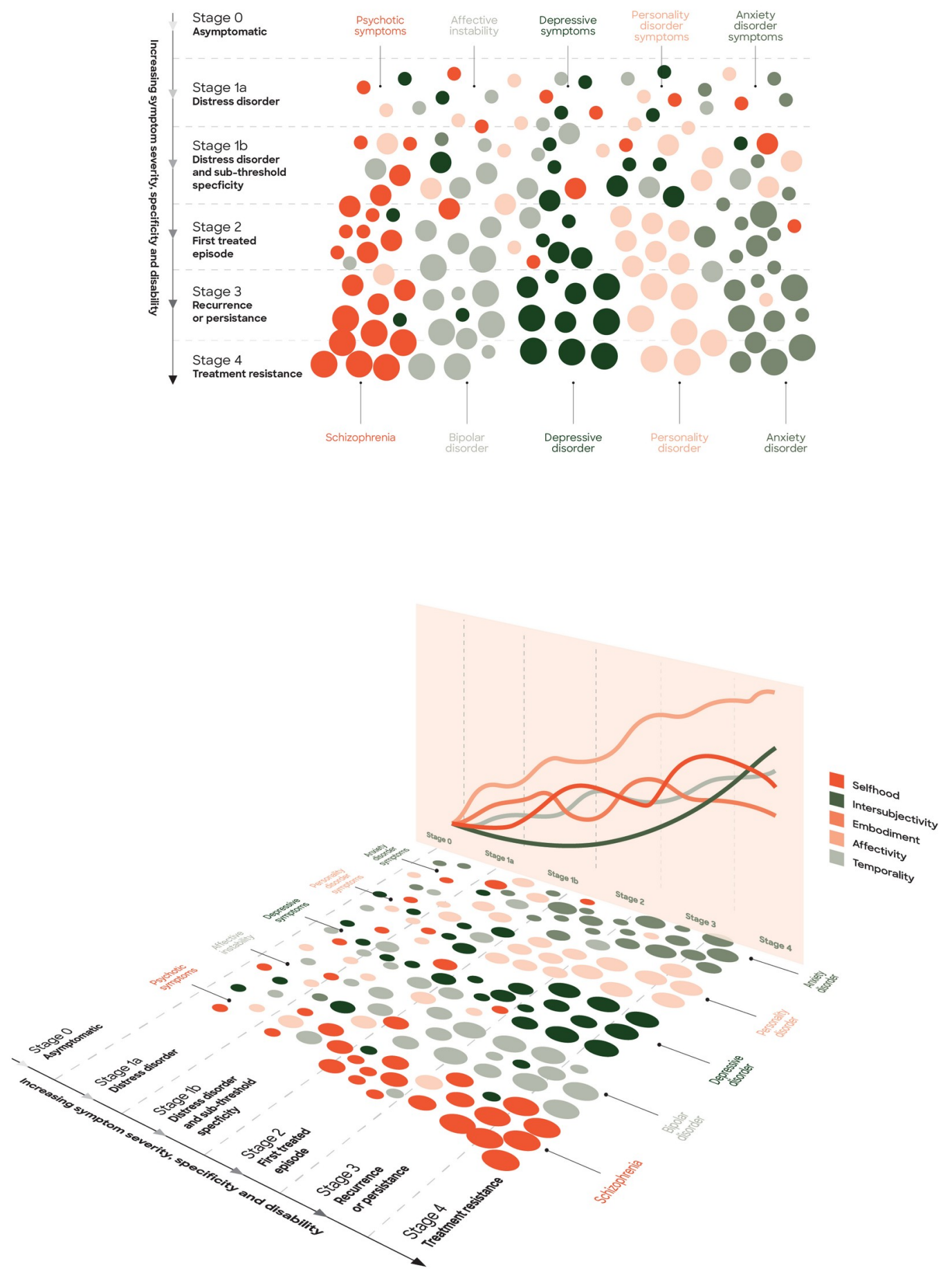
While it may appear that clinical staging and phenomenological psychopathology can prima facie be integrated along these lines, what are the advantages of doing so? We see at least two benefits:

1. A clinical staging-phenomenological psychopathology integration could provide different levels of depth to phenotypic description as required by the clinical or research context (12). For example, assigning clinical stage to a particular patient or group of patients is a useful shorthand in some clinical contexts to succinctly capture illness severity (e.g., "This is a stage 2 clinical presentation" - that is, a first episode of serious mental disorder). This use of clinical staging in fact already occurs in some public mental health services, e.g. at the end of each patient consultation in headspace youth mental health services (27). However, describing disturbances of existential structures (phenomenological psychopathology) would clearly provide greater detail regarding the specific psychopathological disturbances experienced by a patient (i.e., it would enhance phenotypic resolution and depth). In some ways, this may be analogous to supplementing description of presenting complaints with identification of particular underlying beliefs (a psychological formulation) and/or results of biomedical tests (a medical formulation); however, the emphasis here is on detailed characterisation of the clinical phenotype rather than on identifying potential causal contributors to this phenotype. See Table 1 for an example - in this case, the clinical characterisation of a patient at three levels of detail: 1. Stage 2 disorder, 2. Severe positive psychotic symptoms and mild depressed mood, 3. Basic selfdisturbances, disturbed temporality and intersubjectivity.

This 'high resolution' approach capturing clinical stage and psychopathological detail may also be useful in other contexts, such as the development of detailed case 
formulations or construction of treatments plans. For example, a patient with stage 3 disorder characterised by persistently severe depressed mood and disturbances of intersubjectivity may point towards the clinical relevance of disrupted interpersonal attachments and neglect during developmental years (predisposing him/her to intersubjectivity disturbances) and difficulty forming a consistent relationship with a treating team (a factor that has contributed to stage progression by reducing exposure to treatment). Another example might be a treatment plan for a patient at stage $1 \mathrm{~b}$ with attenuated psychotic symptoms and substance dependence with prominent basic self-disturbances that consists of comprehensive psychosocial treatment and substance detoxification and rehabilitation (tailoring treatment to stage of disorder), combined with treatment elements that target the particular experiential disturbances characteristic of basic self-disturbance, such as fostering robust self-presence through body-oriented strategies, immersive activity and physical exercise, and 'talking therapy' that emphasises a shift in the overall framework of altered experience rather than on specific contents of cognition $(28,29)$.

\section{Table 1}

Heuristic of how diagnosis might be summarised to integrate clinical stage with phenomenological phenotype

\begin{tabular}{|c|c|c|}
\hline $\begin{array}{l}\text { Diagnostic } \\
\text { detail }\end{array}$ & Level of diagnostic information & Example \\
\hline 1 & Stage of disorder & Stage 2 \\
\hline 2 & Symptoms/signs & $\begin{array}{ll}\text { - } & \text { Severe acute positive symptoms } \\
\text { - } & \text { Mild depressed mood }\end{array}$ \\
\hline 3 & $\begin{array}{l}\text { Phenomenological disturbances } \\
\text { ('existential structures') }\end{array}$ & $\begin{array}{ll} & \text { Basic self-disturbances } \\
\text { - } & \text { Disturbed temporality } \\
\text { - } & \text { Disturbance of intersubjectivity }\end{array}$ \\
\hline
\end{tabular}

2.

clinical staging-phenomenological psychopathology integration can be conducted both on an idiographic and a nomothetic level. That is, stage of disorder and 
disturbances of existential structures might be captured for a particular patient, as illustrated in the clinical scenarios outlined above. However, the integration might also apply at a syndromal or disorder level, possibly introducing a useful framework for research studies. A researcher might ask a phenotypic research question guided by this integrated approach, i.e., whether a certain combination of illness stage and existential structure disturbances are associated with a particular cluster of symptoms (e.g., Is stage 2 disorder in combination with prominent disturbances of selfhood and embodiment associated with first rank psychotic symptoms?). Psychological or neurocognitive hypotheses regarding symptom aetiology may emerge from this analysis. Equally, the clinical staging-phenomenological psychopathology integrated approach may inform risk stratification studies (e.g., Stage $1 \mathrm{~b}$ patients with prominent disturbances of affectivity and embodiment may be stratified for increased risk for progression to stage 2 mood disorder) and mechanism-focused studies (e.g., Are certain neural circuit dysfunctions associated with a particular combination of illness stage and experiential disturbances?). While phenomenological phenotypes and clinical staging have both already been used independently to guide and organise biomarker and mechanism-focused studies (30-41) the integration of these approaches may maximise these efforts. Biomarkers and mechanisms may not only vary by clinical stage or disturbance of particular existential structures, but by a combination of the two. For example, we recently found that the phenomenological phenotype of basic self-disturbance and neurocognitive and neurophysiological measures of source monitoring disturbances varied by stage of psychotic disorder (becoming more severe as disorder progressed) and that the correlation between these two levels of analysis also became stronger as disorder progressed (i.e., there 
was an interaction between stage of disorder, phenomenological phenotype and neural correlate)(30, 31).

There are clearly a number of challenges that would need to be overcome for the integrative approach outlined above and we are not proposing that this approach is ready for 'primetime'. First, there is the issue of sufficient awareness of and training in phenomenologically-informed assessment of psychopathology. As has been observed by a number of authors [e.g. see (42-44)], this is a weakness in many psychiatry/clinical psychology training programs at present. A second, related issue is that of inter-rater reliability. How reliably can these more detailed, nuanced assessment approaches be implemented? The data to date are actually quite encouraging on this front. For example, the EASE, EAWE and the Bonn Scale for the Assessment of Psychopathology (BSABS) have all demonstrated moderate to strong $(0.6-1.0)$ single item reliability coefficients $(19,20,45)$, certainly comparable to commonly used clinical assessment instruments such as the Brief Psychiatric Rating Scale [BPRS $(46,47)]$ or the Social and Occupational Functioning Scale [SOFAS (48)], and far superior to structured diagnostic interviews conducted by non-clinicians (49). These reliability statistics have been achieved in well-trained research-oriented settings. Perhaps the question should not so much be how these 'high resolution' assessments can be transferred to everyday clinical settings, but how we might ensure that sophisticated training in psychopathology is widely delivered and accurate identification tools are deployed for screening and referral to specialist centres for further assessment and diagnostic clarification when required. A final challenge, related to the other two, is the risk that the proposed integrated approach results in 
complexity that is unwieldy in clinical settings. Rather than prematurely foreclose exploration of the model due to this risk, we propose testing it first in specialised settings, such as early intervention services.

\section{Conclusion}

In this opinion piece, we have considered points of intersection between clinical staging and phenomenological psychopathology's perspective on clinical phenotyping. Domains identified in phenomenological psychopathology, such as selfhood, embodiment, affectivity, etc., can be overlaid on clinical stages in order to enrich and deepen the phenotypes captured in clinical staging ('high resolution' clinical phenotypes). This may be of value for complementing diagnosis, enriching clinical formulation, and informing treatment of individual patients. It may also help guide aetiological, prediction, and treatment research. The overlaying of phenomenological domains on clinical stages may require that these domains are reformulated in dimensional rather than categorial terms. This integrative project requires assessment tools that are sensitive and thorough enough to pick up on the range of relevant psychopathology. Some of these instruments are already available, such as the EASE, EAWE, BSABS and AMDP System: Manual for Assessment and Documentation of Psychopathology in Psychiatry. The overlaying of continuum-based phenomenological concepts on clinical stages also needs to be approached in a careful manner that does not 'break apart' Gestalt concepts such as self- or worlddisturbance, which rely on a certain indivisible relationship between parts (individual symptoms/signs) and the whole (overall structural shift in experience/expression), into atomistic elements that lose their psychopathological importance in the process [see Appendix, p.1]. The integration we propose offers opportunities for mutual 
enrichment: clinical staging may be enriched by introducing greater depth to phenotypes; phenomenological psychopathology may be enriched by introducing stages of severity and disorder progression to phenomenological analysis. 


\section{References}

1. Maj M. Beyond diagnosis in psychiatric practice. Ann Gen Psychiatry. 2020;19:27.

2. McGorry PD. The next stage for diagnosis: validity through utility. World Psychiatry. 2013;12(3):213-5.

3. McGorry PD, Hartmann JA, Spooner R, Nelson B. Beyond the "at risk mental state" concept: transitioning to transdiagnostic psychiatry. World Psychiatry. 2018;17(2):133-42.

4. McGorry PD, Hickie IB, Yung AR, Pantelis C, Jackson HJ. Clinical staging of psychiatric disorders: a heuristic framework for choosing earlier, safer and more effective interventions. Aust N Z J Psychiatry. 2006;40(8):616-22.

5. McGorry PD. Issues for DSM-V: clinical staging: a heuristic pathway to valid nosology and safer, more effective treatment in psychiatry. Am J Psychiatry. 2007;164(6):859-60.

6. Hartmann JA, Nelson B, Spooner R, et al. Broad clinical high-risk mental state (CHARMS): Methodology of a cohort study validating criteria for pluripotent risk. Early Interv Psychiatry. 2019;13(3):379-86.

7. Purcell R, Jorm AF, Hickie IB, et al. Transitions Study of predictors of illness progression in young people with mental ill health: study methodology. Early Interv Psychiatry. 2015;9(1):38-47.

8. Addington J, Liu L, Goldstein BI, et al. Clinical staging for youth at-risk for serious mental illness. Early Interv Psychiatry. 2019;13(6):1416-23.

9. Iorfino F, Scott EM, Carpenter JS, et al. Clinical Stage Transitions in Persons Aged 12 to 25 Years Presenting to Early Intervention Mental Health Services With Anxiety, Mood, and Psychotic Disorders. JAMA Psychiatry. 2019.

10. Shah JL, Scott, J., McGorry, P.D., Cross, S.P.M., Keshavan, M., Nelson, B., Wood, S.J., Marwaha, S., Yung, A.R., Scott, E.M., Öngür, D., Conus, P., Henry, C., Hickie, I.B., for the International Working Group on Transdiagnostic Clinical Staging in Youth Mental Health. Transdiagnostic clinical staging in youth mental health: A first international consensus statement. World Psychiatry. 2020;19(2):233-42.

11. Shah JL. Bringing Clinical Staging to Youth Mental Health: From Concept to Operationalization (and Back Again). JAMA Psychiatry. 2019.

12. Nelson B, Hartmann JA, Parnas J. Detail, dynamics and depth: useful correctives for some current research trends. Br J Psychiatry. 2018;212(5):262-4.

13. Stanghellini G, Broome MR, Fernandez AV, Fusar-Poli P, Raballo A, Rosfort R, editors. The Oxford Handbook of Phenomenological Psychopathology. Oxford: Oxford UP; 2019.

14. Fernandez AV. Phenomenology and Dimensional Approaches to Psychiatric Research and Classification. Philosophy, Psychiatry, and Psychology. 2019;26(1):65-75.

15. Fernandez AV, Køster A. On the Subject Matter of Phenomenological Psychopathology. In: Stanghellini G, Broome M, Fernandez AV, Fusar-Poli P, Raballo A, Rosfort R, editors. The Oxford Handbook of Phenomenological Psychopathology. Oxford: Oxford University Press; 2019. p. 191-204.

16. Fernandez AV. The Subject Matter of Phenomenological Research: Existentials, Modes, and Prejudices. Synthese. 2017;194(9):3543-62.

17. Martin W, Gergel T, Owen GS. Manic temporality. Philos Psychol. 2018;32(1):72-97.

18. Fernandez AV. Clarifying a Dimensional Approach to Phenomenological Psychopathology. Philosophy, Psychiatry, and Psychology. 2019;26(1):81-5. 
19. Parnas J, Moller P, Kircher T, et al. EASE: Examination of Anomalous Self-Experience. Psychopathology. 2005;38(5):236-58.

20. Sass L, Pienkos E, Skodlar B, et al. EAWE: Examination of Anomalous World Experience. Psychopathology. 2017;50(1):10-54.

21. Sass L, Pienkos E. Varieties of self experience: A comparative phenomenology of melancholia, mania, and schizophrenia, Part I. Journal of Consciousness Studies. 2013;20:103-30.

22. Ratcliffe M. Varieties of temporal experience in depression. J Med Philos. 2012;37(2):114-38.

23. Raballo A, Monducci E, Ferrara M, Fiori Nastro P, Dario C, group R. Developmental vulnerability to psychosis: Selective aggregation of basic self-disturbance in early onset schizophrenia. Schizophr Res. 2018;201:367-72.

24. Zandersen M, Parnas J. Borderline personality disorder or a disorder within the schizophrenia spectrum? A psychopathological study. World Psychiatry. 2019;18(1):109-10.

25. Zandersen M, Parnas J. Identity Disturbance, Feelings of Emptiness, and the Boundaries of the Schizophrenia Spectrum. Schizophr Bull. 2019;45(1):106-13.

26. Nelson B, Thompson A, Chanen AM, Amminger GP, Yung AR. Is basic self-disturbance in ultra-high risk for psychosis ('prodromal') patients associated with borderline personality pathology? Early Interv Psychiatry. 2013;7(3):306-10.

27. Rickwood DJ, Telford NR, Parker AG, Tanti CJ, McGorry PD. headspace - Australia's innovation in youth mental health: who are the clients and why are they presenting? Med J Aust. 2014;200(2):108-11.

28. Sass L. Three Dangers: Phenomenological Reflections on the Psychotherapy of Psychosis. Psychopathology. 2019;52(2):126-34.

29. Nelson B, Torregrossa L, Thompson A, et al. Improving treatments for psychotic disorders: Beyond cognitive behaviour therapy for psychosis. Psychosis. 2020; In press.

30. Nelson B, Lavoie S, Gaweda L, et al. The neurophenomenology of early psychosis: An integrative empirical study. Conscious Cogn. 2020;77:102845.

31. Nelson B, Lavoie S, Gaweda L, et al. Testing a neurophenomenological model of basic self disturbance in early psychosis. World Psychiatry. 2019;18(1):104-5.

32. Sestito M, Raballo A, Umilta MA, et al. Mirroring the self: testing neurophysiological correlates of disturbed self-experience in schizophrenia spectrum. Psychopathology. 2015;48(3):184-91.

33. Sestito M, Raballo A, Stanghellini G, Gallese V. Editorial: Embodying the Self: Neurophysiological Perspectives on the Psychopathology of Anomalous Bodily Experiences. Front Hum Neurosci. 2017;11:631.

34. Martin B, Wittmann M, Franck N, Cermolacce M, Berna F, Giersch A. Temporal structure of consciousness and minimal self in schizophrenia. Frontiers in psychology. 2014;5:1175.

35. Martin B, Franck N, Cermolacce M, et al. Fragile temporal prediction in patients with schizophrenia is related to minimal self disorders. Sci Rep. 2017;7(1):8278.

36. Martin B, Franck N, Cermolacce M, Coull JT, Giersch A. Minimal Self and Timing Disorders in Schizophrenia: A Case Report. Front Hum Neurosci. 2018;12:132.

37. Schultze-Lutter F, Debbane M, Theodoridou A, et al. Revisiting the Basic Symptom Concept: Toward Translating Risk Symptoms for Psychosis into Neurobiological Targets. Front Psychiatry. 2016;7:9. 
38. Poletti M, Gebhardt E, Raballo A. Corollary Discharge, Self-agency, and the Neurodevelopment of the Psychotic Mind. JAMA Psychiatry. 2017;74(11):1169-70.

39. Bonoldi I, Allen P, Madeira L, et al. Basic Self-Disturbances Related to Reduced Anterior Cingulate Volume in Subjects at Ultra-High Risk for Psychosis. Front Psychiatry. 2019;10:254.

40. Wood SJ, Yung AR, McGorry PD, Pantelis C. Neuroimaging and treatment evidence for clinical staging in psychotic disorders: from the at-risk mental state to chronic schizophrenia. Biol Psychiatry. 2011;70(7):619-25.

41. McGorry $\mathrm{P}$, Keshavan $\mathrm{M}$, Goldstone $\mathrm{S}$, et al. Biomarkers and clinical staging in psychiatry. World Psychiatry. 2014;13(3):211-23.

42. Andreasen NC. DSM and the death of phenomenology in america: an example of unintended consequences. Schizophr Bull. 2007;33(1):108-12.

43. Mullen PE. A modest proposal for another phenomenological approach to psychopathology. Schizophr Bull. 2007;33(1):113-21.

44. Parnas J, Gallagher S. Phenomenology and the interpretation of psychopathological experience. In: Kirmayer LJ, Leemelson R, Cummings CA, editors. Re-visioning psychiatry: Cultural phenomenology, critical neuroscience and global mental health. New York: Cambridge University Press; 2015. p. 65-80.

45. Vollmer-Larsen A, Handest P, Parnas J. Reliability of measuring anomalous experience: the Bonn Scale for the Assessment of Basic Symptoms. Psychopathology. 2007;40(5):345-8.

46. Flemenbaum A, Zimmermann RL. Inter- and intra-rater reliability of the Brief Psychiatric Rating Scale. Psychol Rep. 1973;32(3):783-92.

47. Bell M, Milstein R, Beam-Goulet J, Lysaker P, Cicchetti D. The Positive and Negative Syndrome Scale and the Brief Psychiatric Rating Scale. Reliability, comparability, and predictive validity. J Nerv Ment Dis. 1992;180(11):723-8.

48. Morosini PL, Magliano L, Brambilla L, Ugolini S, Pioli R. Development, reliability and acceptability of a new version of the DSM-IV Social and Occupational Functioning Assessment Scale (SOFAS) to assess routine social functioning. Acta Psychiatr Scand. 2000;101(4):323-9.

49. Nordgaard J, Revsbech R, Saebye D, Parnas J. Assessing the diagnostic validity of a structured psychiatric interview in a first-admission hospital sample. World Psychiatry. 2012;11(3):181-5. 